\title{
SYMPLECTIC EMBEDDINGS OF 4-DIMENSIONAL ELLIPSOIDS
}

\author{
DUSA MCDUFF
}

\begin{abstract}
As has been known since the time of Gromov's Nonsqueezing Theorem, symplectic embedding questions lie at the heart of symplectic geometry. This talk will discuss some recent developments concerning the question of when a 4-dimensional ellipsoid can be symplectically embedded in a ball. This problem turns out to have unexpected relations to the properties of continued fractions and exceptional curves in blow ups of the complex projective plane.
\end{abstract}

For Michael Atiyah on his 80th birthday

The standard symplectic structure on $\mathbb{R}^{2 n}$ is:

$$
\omega_{0}=d x_{1} \wedge d x_{2}+d x_{3} \wedge d x_{4}+\cdots+d x_{2 n-1} \wedge d x_{2 n} .
$$

By Darboux's theorem every symplectic form is locally diffeomorphic to this one, so it is crucial to understand its properties.

Gromov's question: What are the images of a symplectic ball? How are the symplectic and volume preserving cases different?

Let $B:=B^{2 n}(a)$ be the standard (closed) ball of radius $\sqrt{a}$ (so $a$ is proportional to a 2-dimensional area) and let $\phi: B \stackrel{s}{\hookrightarrow} \mathbb{R}^{2 n}$ be a symplectic embedding (i.e. a smooth embedding onto $\phi(B)$ that preserves $\omega_{0}$ ).

The problem is to describe $\phi(B)$ - for example: can it be long and thin?

Since $\omega_{0}^{n}=n ! d x_{1} \wedge \cdots \wedge d x_{2 n}$ is a volume form, every symplectic embedding preserves volume.

Date: Notes for a talk at Atiyah80, Edinburgh, April 2009. partially supported by NSF grant DMS 0604769. 


\section{Volume preserving embeddings: if $V \subset \mathbb{R}^{2 n}$ is} diffeomorphic to a ball and $\operatorname{vol} V=\operatorname{vol} B$ then there is a volume preserving diffeomorphism $\psi: B \rightarrow V$.

The analogous statement is NOT true for symplectomorphisms since the boundary of $V$ has symplectic invariants. Nevertheless one might ask if one can fully fill $V$ by a symplectic ball. This means that for every $\varepsilon>0$ there is a ball $B$ and a symplectic embedding $\phi: B \stackrel{s}{\hookrightarrow} V$ such that $\operatorname{vol}(V \backslash \phi(B))<\varepsilon$.

Let $Z(A)$ be the cylinder

$$
D^{2}(A) \times \mathbb{R}^{2 n-2}=\left\{\left(x_{1}, \ldots, x_{2 n}\right): x_{1}^{2}+x_{2}^{2} \leq A\right\}
$$

\section{Gromov's Nonsqueezing Theorem: There is a} symplectic embedding $\phi: B^{2 n}(a) \stackrel{s}{\hookrightarrow} Z(A)$ if and only if $a \leq A$.

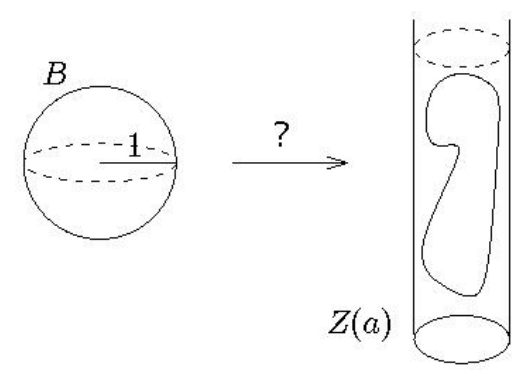

Figure 0.1. Does the ball embed in the cylinder?

[Eliashberg and Hofer]: A diffeomorphism that has this nonsqueezing property for all balls and all symplectic cylinders must preserve the symplectic structure. 
Embedding disjoint unions of balls. How much of the volume of $B(1)$ can be filled by $k$ equal symplectic balls?

[Gromov]: there are obstructions when $k=2$ :

$$
\text { if } B(a) \sqcup B(a) \stackrel{s}{\hookrightarrow} B(1) \text { then } a \leq 1 / 2 \text {; }
$$

i.e. the volume of the image of $B(a) \sqcup B(a)$ is $\leq \frac{1}{2^{n-1}} \operatorname{vol}(B(1))$.

[McDuff and Polterovich]: for any $d \geq 1$ the $2 n$-dimensional ball $B(1)$ can be fully filled by $k=d^{n}$ equal balls.

\section{Describing embeddings by toric models:}

Consider the (moment) map $\Phi: \quad \mathbb{C}^{2} \rightarrow \mathbb{R}^{2}, \Phi\left(z_{1}, z_{2}\right)=\left(\left|z_{1}\right|^{2},\left|z_{2}\right|^{2}\right)$. Then $\Phi(B(1))$ is the (standard) triangle $\left\{0 \leq x_{1}, x_{2} ; x_{1}+x_{2} \leq 1\right\}$. Since every such triangle can be fully filled by a ball, we can see the full fillings of $B^{4}(1)$ by $d^{2}$ equal balls.
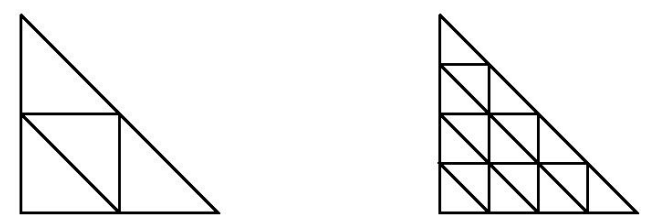

FiguRE 0.2 . Embedding $d^{2}$ standard triangles into a triangle of size 1 .

[McDuff-Polterovich]: In dimension 4 there are obstructions when $k<9, k \neq 1,4$;

[Biran]: $B^{4}(1)$ can be fully filled by $k$ equal balls for all $k \geq 9$ i.e. with enough balls, the obstructions disappear.

From now on we work in 4 (real) dimensions, and $E \stackrel{s}{\hookrightarrow} B$ means there is a symplectic embedding of $E$ into $B$. 


\section{Embedding ellipsoids into balls}

Let $E(a, 1)=\left\{\left(x_{1}, \ldots, x_{4}\right) \in \mathbb{R}^{4}: \frac{x_{1}^{2}+x_{2}^{2}}{a}+x_{3}^{2}+x_{4}^{2} \leq 1\right\}$.

Consider the embedding capacity function of [2] for $a \geq 1$ :

$$
c(a):=\inf \{\mu: E(a, 1) \stackrel{s}{\hookrightarrow} B(\mu)\} .
$$

Note: $c(a) \geq \sqrt{a}$ because vol $E(a, 1)=\operatorname{vol} B(\sqrt{a})$.

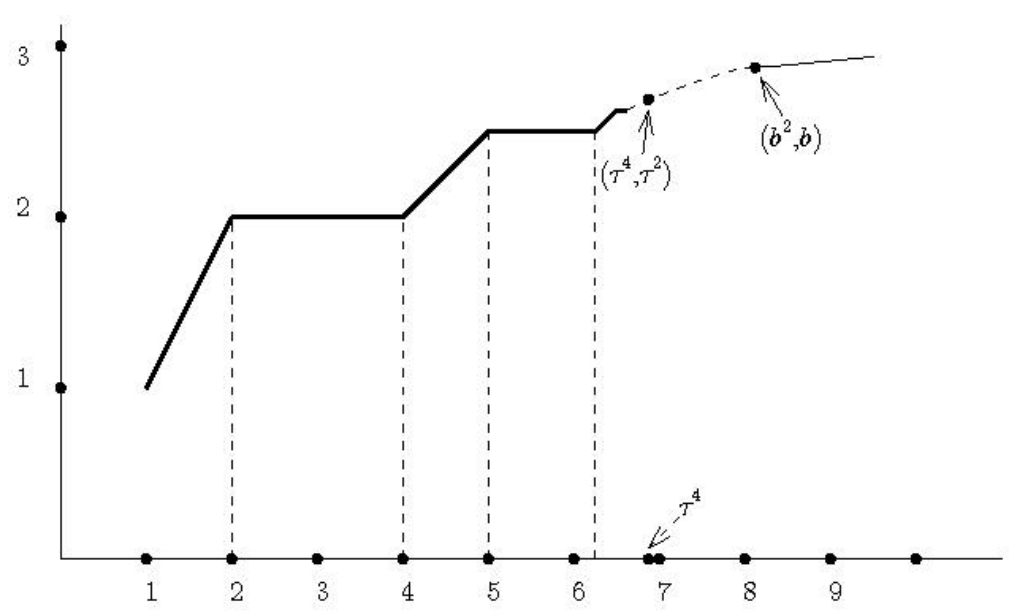

Figure 0.3. The graph of $c(a)$

Theorem [McDuff-Schlenk] Let $\tau=\frac{1+\sqrt{5}}{2}$. The graph of $c(a)$ divides into three parts:

- if $1 \leq a<\tau^{4}$ the graph is piecewise linear - an infinite Fibonacci staircase converging to $\left(\tau^{4}, \tau^{2}\right)$; (mostly proven)

- $\tau^{4} \leq a<8 \frac{1}{36}$ is a transitional region; $c(a)=\sqrt{a}$ except on a finite number of short intervals; (mostly proven)

- if $a \geq 8 \frac{1}{36}=\left(\frac{17}{6}\right)^{2}$ then $c(a)=\sqrt{a}$. (proven)

Fibonacci stairs: Let $g_{0}=g_{1}=1, g_{2}=2, g_{3}=5, g_{4}=13, \ldots$, the odd Fibonacci numbers; set $a_{n}:=\left(g_{n+1} / g_{n}\right)^{2}, \quad b_{n}:=g_{n+2} / g_{n}$ so that $a_{n}<b_{n}<a_{n+1}$, and $a_{n} \rightarrow \tau^{4}$.

Then $c(x)=x / \sqrt{a_{n}}$ on $\left[a_{n}, b_{n}\right]$ and $c(x)=\sqrt{a_{n+1}}$ on $\left[b_{n}, a_{n+1}\right]$. 
Let $\boldsymbol{w}(a)=\left(w_{1}, \ldots, w_{N}\right)$ be the weight expansion of $a \in \mathbb{Q}$ :

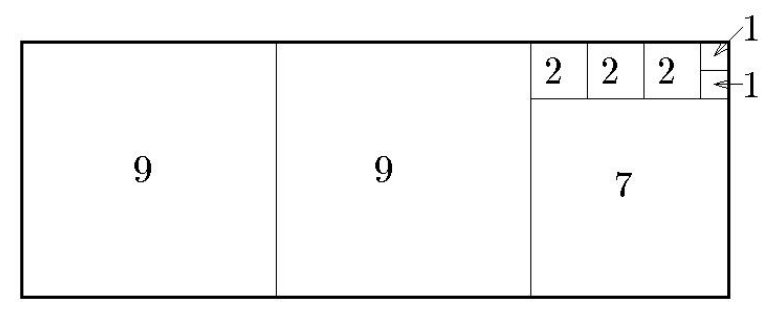

Figure 0.4. $\boldsymbol{w}(25 / 9)=(1,1,7 / 9,2 / 9,2 / 9,2 / 9,1 / 9,1 / 9)$. The multiplicities $2,1,3,2$ of the weights are the terms (or partial quotients) of the continued fraction $[2 ; 1,3,2]$ representation of $25 / 9$.

Thm I $[\mathrm{McD}] E(a, 1) \stackrel{s}{\hookrightarrow} B(\mu) \Longleftrightarrow \underset{i \leq N}{\bigsqcup_{\leq}} B\left(w_{i}\right) \stackrel{s}{\hookrightarrow} B(\mu)$.

Given $N$ write $\boldsymbol{m}:=\left(m_{1}, \ldots, m_{N}\right) \in \mathbb{N}^{N}, m_{i} \geq m_{i+1}$. Let

$$
\mathcal{E}_{N}=\left\{(d ; \boldsymbol{m}): d^{2}+1=\sum_{i} m_{i}^{2}, 3 d-1=\sum_{i} m_{i},\right.
$$

and another algebraic condition $\}$.

Then $\mathcal{E}_{N}=$ the set of homology classes $d L-\sum m_{i} E_{i}$ represented by symplectic exceptional divisors in the $N$ fold blow up of $\mathbb{C} P^{2}$.

Thm II [McD-Polt, Biran, Li-Li]

$$
\bigsqcup_{i \leq N} B\left(w_{i}\right) \stackrel{s}{\hookrightarrow} B(\mu) \Longleftrightarrow \mu d \geq \sum m_{i} w_{i}
$$

for all $(d ; \boldsymbol{m}) \in \mathcal{E}_{N}$.

Thus $c(a)=\sup \left\{\sqrt{a}, \sup _{(d, \boldsymbol{m}) \in \mathcal{E}_{N}} \frac{\sum m_{i} w_{i}(a)}{d}\right\}$.

Example: $\mathcal{E}_{4}$ has the single element $(1 ; 1,1)$ (corresponding to $\left.L-E_{1}-E_{2}\right)$ and $\boldsymbol{w}(4)=(1,1,1,1)$. Therefore $c(4)=2$.

$\mathcal{E}_{5}$ also contains $(2 ; 1, \ldots, 1)$ corresponding to $2 L-\sum_{i=1}^{5} E_{i}$. Thus $c(5)=\sup \{\sqrt{5}, 2,5 / 2\}=5 / 2$. 
Fact: If $a=b_{n}=g_{n+2} / g_{n}$ then $\left(g_{n+1} ; g_{n} \boldsymbol{w}(a)\right) \in \mathcal{E}_{N}$.

Example: $n=2$ gives: $(5 ; 2 \boldsymbol{w}(13 / 2))=(5 ; 2, \ldots, 2,1,1)$ and $5 L-2\left(E_{1}+\cdots+E_{6}\right)-E_{7}-E_{8} \in \mathcal{E}_{8}$.

Using this, we can find $c\left(b_{n}\right)$ for all $n$ :

$$
\begin{aligned}
d \mu \geq \sum m_{i} w_{i} & \Longrightarrow g_{n+1} \mu \geq g_{n} \sum w_{i}^{2}=g_{n} a \\
& \Longrightarrow \mu \geq\left(g_{n} g_{n+2}\right) /\left(g_{n+1} g_{n}\right)=\sqrt{a_{n+1}} .
\end{aligned}
$$

This shows $c\left(b_{n}\right) \geq \sqrt{a_{n+1}}$. We get $=$ because $d d^{\prime} \geq \sum m_{i} m_{i}^{\prime}$ for all distinct pairs $(d, \boldsymbol{m}),\left(d^{\prime}, \boldsymbol{m}^{\prime}\right) \in \mathcal{E}_{N}$ (positivity of intersections).

Conjecture: If $a=\left(g_{n+1} / g_{n}\right)^{2}$ then $\left(g_{n} g_{n+1}, g_{n}^{2}(\boldsymbol{w}(a), 1)\right) \in \mathcal{E}_{N}$.

(Still need to establish the third condition; if the conjecture holds, the stairs are proven.)

\section{What is special about $a=\tau^{4}$ ?}

$a=\tau^{4}$ is the positive root of $3 \sqrt{a}=a+1$. The sharpest constraints come from $(d, \boldsymbol{m})$ where $\boldsymbol{m} \approx \lambda \boldsymbol{w}(a)$. Since $\sum w_{i}^{2}=a$ and $\sum m_{i}^{2} \approx$ $d^{2}$, we need $\lambda \approx d / \sqrt{a}$. But also

$$
\sum w_{i}=1+a-1 / q \approx 1+a, \quad \sum \lambda w_{i} \approx \sum_{i} m_{i} \approx 3 d
$$

implies $3 d \approx \lambda(1+a)$ or $3 \sqrt{a} \approx 1+a$.

Why there are no constraints for $a>9$.

We saw above that $c(a)=\sup _{(d, \boldsymbol{m}) \in \mathcal{E}} \frac{\sum m_{i} w_{i}(a)}{d}$. Hence $c(a)>\sqrt{a}$ only if there is $(d, \boldsymbol{m}) \in \mathcal{E}$ such that $\sum m_{i} w_{i}(a) / d>\sqrt{a}$. But $\sqrt{a} \geq 3$, and

$$
\sum m_{i} w_{i} \leq \sum m_{i}=3 d-1 \quad \text { since all } w_{i} \leq 1 .
$$

So $\sum m_{i} w_{i} / d \leq 3$ always. More complicated versions of this argument show there are no constraints for $a \geq 8 \frac{1}{36}$. 
Ideas behind the Proof:

\section{I: Embedding balls and blowing up:}

To blow up a point in complex geometry you remove the point and replace it by the set of all lines through that point. In 2 complex dimensions, this set is a complex line $\mathbb{C} P^{1}$ called the exceptional divisor $E \cong \mathbb{C} P^{1}$. Its normal bundle $\pi: L \rightarrow E$ has Chern class -1 .

Basic fact: $L \backslash E \cong \mathbb{C}^{2} \backslash\{0\}$.

In symplectic geometry, by Darboux's theorem you can identify a nbhd of a point $p$ in $(M, \omega)$ with a nbhd of $\{0\}$ in $\mathbb{R}^{4} \equiv \mathbb{C}^{2}$, and blow up as before to get a manifold $\widetilde{M}$ with blow down map $b l: \widetilde{M} \rightarrow M$. But you must put a symplectic form on $\widetilde{M}$, and the pullback form $b l^{*}(\omega)$ vanishes on $E$. So the form $\widetilde{\omega}_{a}$ on $\widetilde{M}$ equals $b l^{*}(\omega)+a \pi^{*}\left(\sigma_{E}\right)$ near $E$, where $\int_{E} \sigma_{E}=1$ and $\pi: \operatorname{nbhd}(E) \rightarrow E$.

Basic fact: $\left((n b h d E \backslash E), \widetilde{\omega}_{a}\right)$ is symplectomorphic to $\mathbb{C}^{2} \backslash B(a)$. i.e. To blow up symplectically with weight $a$ you remove an embedded copy of the open ball int $B(a)$ and collapse $\partial B(a)=S^{3}$ to $S^{2} \equiv E$ by the Hopf map.

\section{II: Cutting $E(a, 1)$ into balls via toric models}

If $a=p / q$ the image of $E(a, 1)$ under $\Phi: \mathbb{C}^{2} \rightarrow \mathbb{R}^{2},\left(z_{1}, z_{2}\right) \mapsto$ $\left(\left|z_{1}\right|^{2},\left|z_{2}\right|^{2}\right)$ is the triangle $\left\{0 \leq x_{1}, x_{2} ; q x_{1}+p x_{2} \leq 1\right\}$. This corresponds to a singular variety, with singular points of orders $p, q$. We can cut this triangle into standard triangles of different sizes.
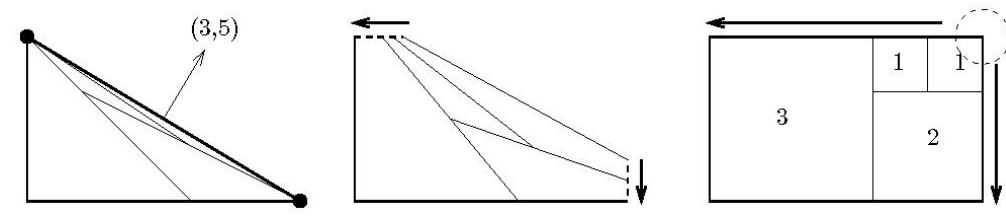

The triangle decomposition is structurally the same as that giving the weights for $5 / 3$. It corresponds to a joint resolution of the two singularities. 

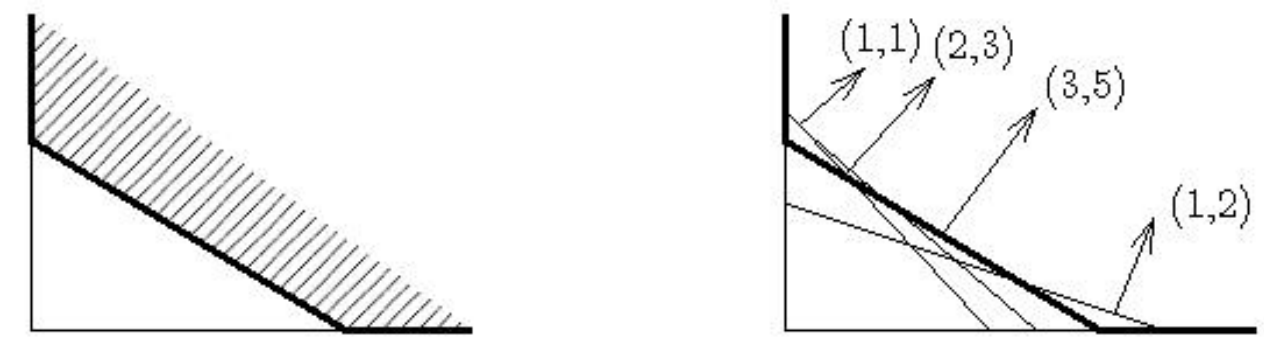

The cuts needed to resolve the two singularities in the shaded polytope on the left (after the triangle is removed) are illustrated on the right: they are parallel to those that decomposed the singular triangle into standard triangles: for details see [5].

If $p>2 q$ the weight decomposition for $p / q$, corresponding to Euclidean continued fractions (with + signs), is related to the multiplicities of the two singular points, which are given by the HirzebruchJung (HJ) continued fractions (with - signs) for $p / q$ and $p /(p-q)$. This relation is captured in the Riemenschneider staircase:

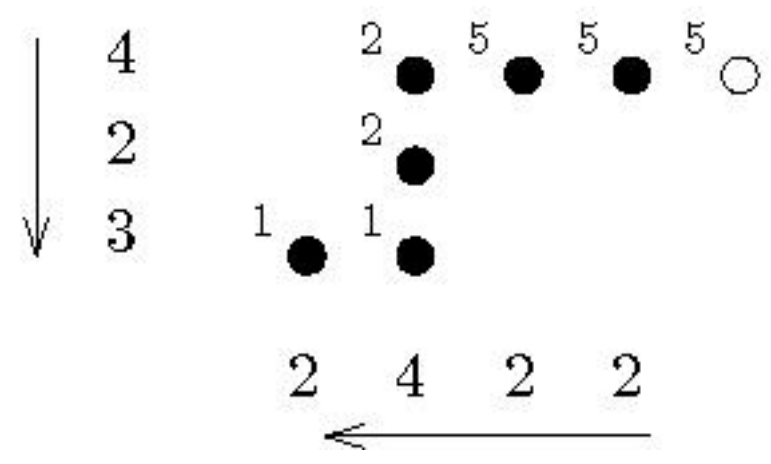

Here, the dots are labelled starting at the top right with the (normalized) weight decomposition $(5,5,5,2,2,1,1)$ for $17 / 5$. The digits 4,2,3 are one more than the number of black dots in the each row, and give the HJ multiplicities for $17 / 5$. The digits 2,2,4,2 are one more than the numbers of black dots in the columns, and give the HJ multiplicities for $17 /(17-5)=17 / 12$. Thus

$$
\frac{17}{5}=\mathbf{3}+\frac{1}{\mathbf{2}+\frac{1}{2}}=\mathbf{4}-\frac{1}{2-\frac{1}{3}}, \quad \frac{17}{12}=\mathbf{2}-\frac{1}{\mathbf{2}-\frac{1}{4-\frac{1}{2}}} .
$$




\section{REFERENCES}

[1] P. Biran, Symplectic packing in dimension 4, Geometric and Functional Analysis, 7 (1997), 420-37.

[2] K. Cieliebak, H. Hofer, J. Latschev and F. Schlenk, Quantitative symplectic geometry, arXiv:math/0506191, Dynamics, Ergodic Theory, Geometry MSRI, 54 (2007), 1-44.

[3] M. Gromov, Pseudo holomorphic curves in symplectic manifolds, Inventiones Mathematicae, 82 (1985), $307-47$.

[4] Bang-He Li and T.-J. Li, Symplectic genus, minimal genus and diffeomorphisms, Asian J. Math. 6 (2002), $123-44$.

[5] D. McDuff, Symplectic embeddings of 4-dimensional ellipsoids, to appear in Journ. of Top..

[6] D. McDuff and L. Polterovich, Symplectic packings and algebraic geometry, Inventiones Mathematicae, 115 (1994), $405-29$.

[7] D. McDuff and F. Schlenk, The embedding capacity of 4-dimensional symplectic ellipsoids, in preparation.

[8] E. Opshtein, Maximal symplectic packings of $\mathbb{P}^{2}$, arxiv:0610677.

Department of Mathematics, Barnard College, Columbia University, New York, Ny 10027-6598, USA.

E-mail address: dmcduff@barnard.edu 